\title{
Editorial
}

\section{Inverse Fluid Convection Problems in Enclosures}

\author{
Fu-Yun Zhao, ${ }^{1,2}$ Di Liu, ${ }^{3}$ and Steve H. L. Yim ${ }^{4}$ \\ ${ }^{1}$ Faculty of Civil Engineering and Geodesy, Technical University Munich, Arcisstr. 21, \\ 80333 Munich, Germany \\ ${ }^{2}$ School of Power and Mechanical Engineering, Wuhan University, Luo-Jia-Shan, \\ Wuchang 430072, China \\ ${ }^{3}$ College of Pipeline and Civil Engineering, China University of Petroleum, Changjiang Road 66, \\ Qingdaos 266580, China \\ ${ }^{4}$ Department of Aeronautics and Astronautics, Massachusetts Institute of Technology, \\ Massachusetts Avenue 77, Cambridge, MA 02139, USA \\ Correspondence should be addressed to Fu-Yun Zhao, zfycfdnet@163.com
}

Received 20 November 2012; Accepted 20 November 2012

Copyright (c) 2012 Fu-Yun Zhao et al. This is an open access article distributed under the Creative Commons Attribution License, which permits unrestricted use, distribution, and reproduction in any medium, provided the original work is properly cited.

Efficiency, security, and reliability of industrial and domestic processes essentially depend on the deep understanding of their actual processes of fluid flow and heat transfer. Actual processes of fluid flow control and measurements need the development of effect-cause inverse modeling. Extensive investigations on the effect-cause inverse modeling could effectively enhance the efficiency, security, and reliability of these industrial and domestic fluid flow processes.

This special issue on the inverse fluid flow compiles 10 papers, merging from different academic disciplines and engineering application backgrounds. Three branches of inverse modeling and application have been discussed. These include inverse identification of pollutant sources, inverse determination of boundary conditions, and inverse measurement of heating devices.

Inverse identification of pollutant source location was addressed in the paper by S. Abe et al., who extended the quasi-reversibility methodology into the situation of airborne pollutant dispersions in the urban street canyons. Timely identification of these pollutant sources could facilitate occupants to evacuate from the dangerous areas.

Unknown boundary conditions always pose the classic modeling of inverse heat transfer and fluid flow. J. Wu et al. employed the hybrid immersed boundary lattice Boltzmann method to determine the unknown heat source term in the immersed boundary, such that the interpolated temperatures accurately satisfy the thermal boundary conditions.

Comparing with the aforementioned theoretical identifications of unknown boundary condition or source information, monitoring and measurement on the transport processes 
could involve the noise data and many uncertainties. C. Solisio et al. applied Tikhonov regularization rules to successfully reconstruct the temperature profiles in the landfills of bioreactor with heat recovery fluids measurements.

In addition to the research papers on the inverse modeling and applications, a few papers concerning the direct modeling of industrial multiphase flows, hydrogen-air detonation, square cylinder flows, and convergent channel flows have also been included in this issue. Outlook optimization and process control on these complicated flows will be investigated in the future.

By compiling these papers, we expect that this special issue on inverse flow problems in the industrial and domestic processes could enrich our readers and researchers.

Fu-Yun Zhao

Di Liu

Steve H. L. Yim 


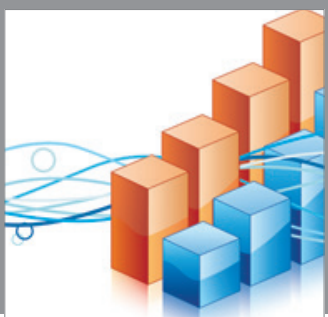

Advances in

Operations Research

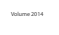

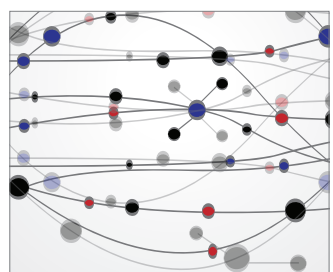

\section{The Scientific} World Journal
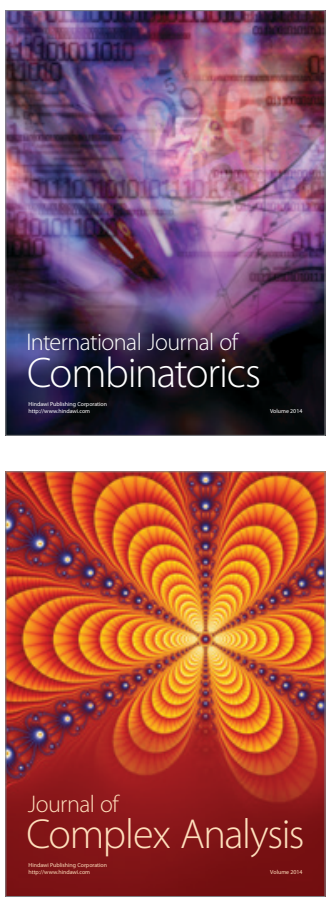

International Journal of

Mathematics and

Mathematical

Sciences
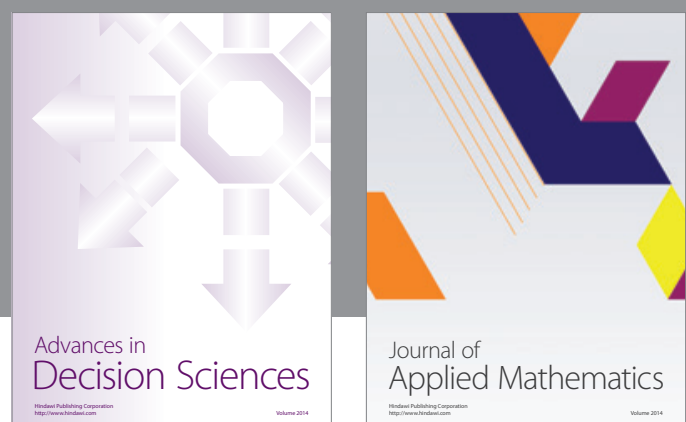

Journal of

Applied Mathematics
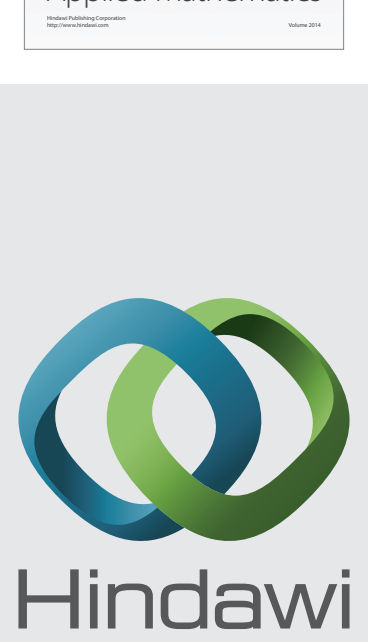

Submit your manuscripts at http://www.hindawi.com
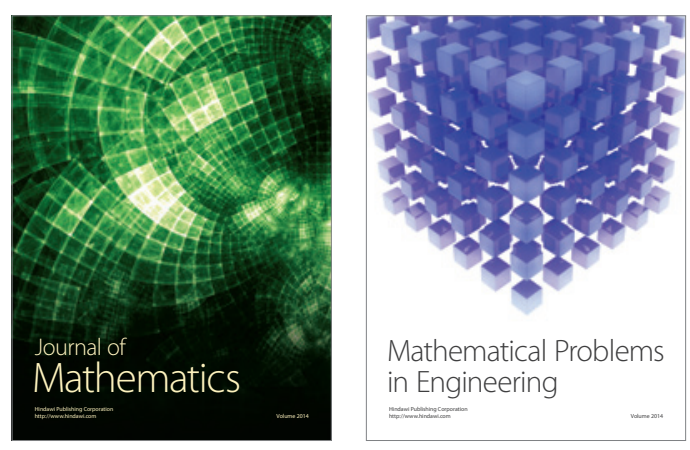

Mathematical Problems in Engineering
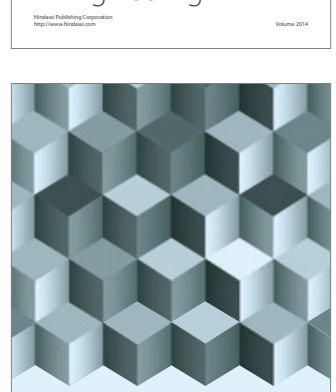

Journal of

Function Spaces
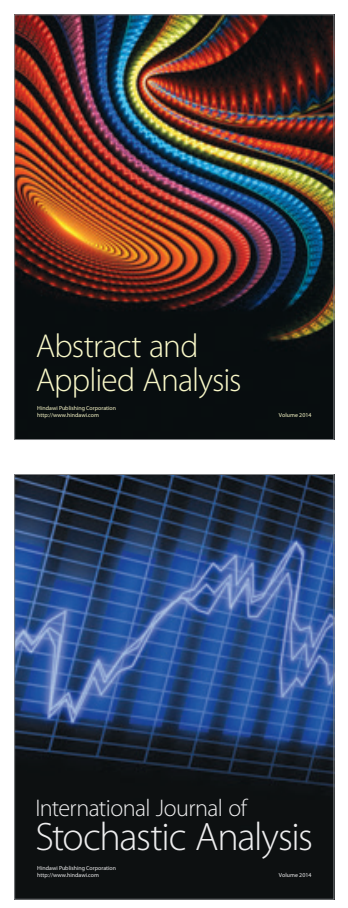

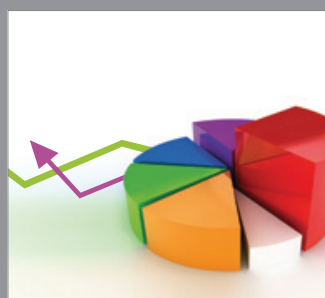

ournal of

Probability and Statistics

Promensencen
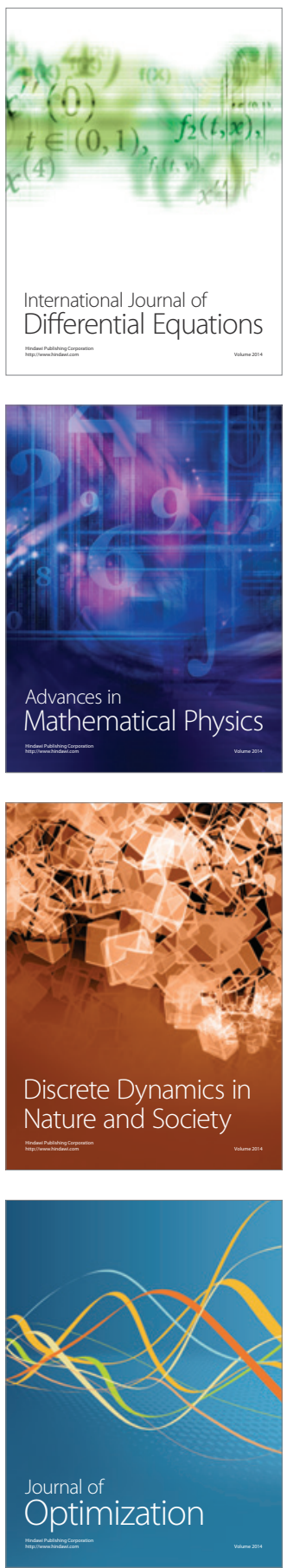